\title{
Foreword from the IMIA President
}

\section{N. M. Lorenzi}

Vanderbilt University Medical Center, Informatics Center, Eskind Biomedical Library, Biomedical Informatics, Nashville, TN, USA

Dear Yearbook of Medical Informatics Reader,

Welcome to the 2006 edition of the Yearbook of Medical Informatics with its focus on Assessing Information Technologies for Health.

Almost daily we learn about a new technology in health care that can improve the speed and accuracy of diagnosis, alleviate pain for both chronic and acute disease, cure disease, lengthen survival, support rehabilitation and help to monitor and prevent disease. Information systems and technology are at the heart of these new health care technologies. Most information technologies have powerful decision making algorithms at their core and without these health care technology would not be either effective or reliable.

In addition to the health technology informatics based information and communication systems support the rapidly growing health care area in ever increasing ways. Information systems make health services more efficient and effective and improve direct and indirect care whether in cities or in remote areas.

The strength of information systems and technology contribution relies on their

- Establishing and implementing data standards and terminology as a core component of the information system.
- Designing, building, and promoting the adoption of information systems to improve the decision making capability of clinicians and in turn to improve health and health care for individuals.

- By integrating the multiple current systems in ways that are useful to the day-to-day clinical care process.

- By effectively managing the adoption process to ensure successful use of the system.

The 2006 Yearbook of Medical Informatics brings you a comprehensive overview of the multiple strategies that have been used to assess information technology for health care as well as outcomes of information technology use. The contents of this publication have been filtered and re-filtered to bring you the best articles and information possible about the strategies and practices of assessing information technology for health. Multiple reviewers have rated and ranked the items included within this publication. This publication will be an important reference resource for you today and in the future. I hope that this comprehensive reference publication of the International Medical Informatics Association will help you today and in the future.

\section{Sincerely,}

Nancy M. Lorenzi, PhD

IMIA President 2004-2007 